\title{
Criteria for rational smoothness of some symmetric orbit closures
}

\author{
Axel Hultman \\ Department of Mathematics, KTH-Royal Institute of Technology, SE-100 44, Stockholm, Sweden.
}

\begin{abstract}
Let $G$ be a connected reductive linear algebraic group over $\mathbb{C}$ with an involution $\theta$. Denote by $K$ the subgroup of fixed points. In certain cases, the $K$-orbits in the flag variety $G / B$ are indexed by the twisted identities $\iota(\theta)=\left\{\theta\left(w^{-1}\right) w \mid w \in W\right\}$ in the Weyl group $W$. Under this assumption, we establish a criterion for rational smoothness of orbit closures which generalises classical results of Carrell and Peterson for Schubert varieties. That is, whether an orbit closure is rationally smooth at a given point can be determined by examining the degrees in a "Bruhat graph" whose vertices form a subset of $\iota(\theta)$. Moreover, an orbit closure is rationally smooth everywhere if and only if its corresponding interval in the Bruhat order on $\iota(\theta)$ is rank symmetric.

In the special case $K=\mathrm{Sp}_{2 n}(\mathbb{C}), G=\mathrm{SL}_{2 n}(\mathbb{C})$, we strengthen our criterion by showing that only the degree of a single vertex, the "bottom one", needs to be examined. This generalises a result of Deodhar for type $A$ Schubert varieties.

Résumé. Soit $G$ un groupe algébrique connexe réductif sur $\mathbb{C}$, équipé d'une involution $\theta$. Soit $K$ le sous-groupe de ses points fixes. Dans certains cas, les orbites des points de la variété de drapeaux $G / B$ sous l'action de $K$ sont indexées par les identités tordues, $\iota(\theta)=\left\{\theta\left(w^{-1}\right) w \mid w \in W\right\}$, du groupe de Weyl $W$. Sous cette hypothèse, on établit un critère pour la lissité rationnelle des adhérences des orbites, qui généralise des résultats classiques de Carrell et Peterson pour les variétés de Schubert. Plus précisément, on peut déterminer si l'adhérence d'une orbite est rationnellement lisse en examinant les degrés dans un "graphe de Bruhat" dont les sommets forment un sousensemble de $\iota(\theta)$. En outre, l'adhérence d'une orbite est partout rationnellement lisse si et seulement si l'intervalle correspondant dans l'ordre de Bruhat de $\iota(\theta)$ est symétrique respectivement au rang.

Dans le cas particulier $K=\mathrm{Sp}_{2 n}(\mathbb{C}), G=\mathrm{SL}_{2 n}(\mathbb{C})$, nous améliorons notre critère en montrant qu'il suffit d'examiner le degré d'un seul sommet, celui "du bas". Ceci généralise un résultat de Deodhar pour les variétés de Schubert de type $A$.
\end{abstract}

Keywords: Rational smoothness, symmetric orbit, Bruhat graph

\section{Introduction}

Let $G$ be a connected reductive complex linear algebraic group equipped with an automorphism $\theta$ of order 2. There is a $\theta$-stable Borel subgroup $B$ which contains a $\theta$-stable maximal torus $T$ [Ste68, §7] with normaliser $N$. Let $K=G^{\theta}$ be the fixed point subgroup. We may always assume $\theta$ to be the complexification of the Cartan involution of some real form $G_{\mathbb{R}}$ of $G$. 
The flag variety $X=G / B$ decomposes into finitely many orbits under the action of the symmetric subgroup $K$ by left translations. A natural "Bruhat-like" partial order on the set of orbits $K \backslash X$ is defined by inclusion of their closures. Let $V$ denote this poset. Richardson and Springer [RS90, RS94] defined a poset map $\varphi: V \rightarrow \operatorname{Br}(W)$, where $\operatorname{Br}(W)$ is the Bruhat order on the Weyl group $W=N / T$. The image of $\varphi$ is contained in the set of twisted involutions $\mathcal{I}(\theta)=\left\{w \in W \mid \theta(w)=w^{-1}\right\}$. In general, $\varphi$ is neither injective nor surjective. For certain choices of $G$ and $\theta$, however, $\varphi$ produces a poset isomorphism $V \cong \operatorname{Br}(\iota(\theta))$, where $\iota(\theta)=\left\{\theta\left(w^{-1}\right) w \mid w \in W\right\} \subseteq \mathcal{I}(\theta)$ is the set of twisted identities and $\operatorname{Br}(\cdot)$ denotes induced subposet of $\operatorname{Br}(W)$. In Section 3, we shall make explicit under what circumstances this fairly restrictive assumption holds. Now suppose that $\varphi$ is such an isomorphism and let $\overline{\mathcal{O}_{w}}, w \in \iota(\theta)$, denote the closure of the orbit $\mathcal{O}_{w}=\varphi^{-1}(w)$. In this article we express the rationally singular locus of $\overline{\mathcal{O}_{w}}$ in terms of the combinatorics of $\iota(\theta)$.

With each $w \in \iota(\theta)$, we associate a Bruhat graph $\mathrm{BG}(w)$ with vertex set $I_{w}=\{u \in \iota(\theta) \mid u \leq w\}$. Our first main result, Theorem 5.7 states that $\overline{\mathcal{O}_{w}}$ is rationally smooth at $\mathcal{O}_{u}$ if and only if $v$ is contained in $\rho(w)$ edges for all $u \leq v \leq w$, where $\rho(w)$ is the rank of $w$ in $\operatorname{Br}(\iota(\theta))$. In particular, $\overline{\mathcal{O}_{w}}$ is rationally smooth if and only if $\operatorname{BG}(w)$ is $\rho(w)$-regular. This latter statement also turns out to be equivalent to the principal order ideal $\operatorname{Br}\left(I_{w}\right)$ being rank-symmetric; see Theorem 5.8 below.

The assertions just stated generalise celebrated criteria due to Carrell and Peterson [Car94] for rational smoothness of Schubert varieties. We recover their results in the special case where $G=G^{\prime} \times G^{\prime}$ and $\theta(x, y)=(y, x)$.

The main brushstrokes of our proofs are completely similar to those of Carrell and Peterson. Below the surface, however, their results rely on delicate connections between Kazhdan-Lusztig polynomials and the combinatorics of (ordinary) Bruhat graphs. Our chief contribution is to extend these properties to a more general setting. Very roughly, here is what we do:

First, properties of $\iota(\theta)$ are established that combined with results of Brion [Bri99] imply a bound on the degrees in $\mathrm{BG}(w)$ that generalises "Deodhar's inequality" for degrees in ordinary Bruhat graphs of Weyl groups.

Second, an explicit procedure, in terms of combinatorial properties of $\iota(\theta)$, for computing the " $R$ polynomials" of [LV83, $\operatorname{Vog} 83]$ is extracted from the correspondence $V \leftrightarrow \iota(\theta)$. Using this procedure we establish several properties of these polynomials (and therefore of Kazhdan-Lusztig-Vogan polynomials) and relate them to degrees in the graphs $\mathrm{BG}(w)$. This generalises well known properties of ordinary Kazhdan-Lusztig polynomials and $R$-polynomials and how they are related to ordinary Bruhat graphs.

The most prominent example where our results say something which is not contained in [Car94] is $G=\mathrm{SL}_{2 n}(\mathbb{C}), K=\mathrm{Sp}_{2 n}(\mathbb{C})$. For this setting, we prove the stronger statement (Corollary 6.5 that the degree of the bottom vertex alone suffices to decide rational smoothness. That is, $\overline{\mathcal{O}_{w}}$ is rationally smooth at $\mathcal{O}_{u}$ if and only if the degree of $u$ in $\operatorname{BG}(w)$ is $\rho(w)$. This is analogous to a corresponding result for type $A$ Schubert varieties which is due to Deodhar [De085]. Again, that result is contained in ours as a special case.

Remark 1.1 After a preliminary version of [Hul09] was circulated, McGovern [McG09] has applied our results in order to deduce a criterion for (rational) smoothness in the case $G=\mathrm{SL}_{2 n}(\mathbb{C}), K=\mathrm{Sp}_{2 n}(\mathbb{C})$ in terms of pattern avoidance among fixed point free involutions. Moreover, he proved that in this case the rationally singular loci in fact coincide with the singular loci.

In Section 3, we make precise the assumptions on $\theta$ for which our results are valid. Thereafter, the Bruhat graphs BG(w) are introduced in Section 4 . Our Carrell-Peterson type criteria for rational smooth- 
ness are deduced in Section 5. Finally, in Section 6, we prove that the bottom vertex alone suffices to decide rational smoothness when $G=\mathrm{SL}_{2 n}(\mathbb{C}), K=\mathrm{Sp}_{2 n}(\mathbb{C})$.

Details left out in the present extended abstract can be found in [Hul09].

\section{Kazhdan-Lusztig-Vogan polynomials}

In the present paper, the principal method for detecting rational singularities of symmetric orbit closures is via Kazhdan-Lusztig-Vogan polynomials. Here, we briefly review some of their properties and establish notation. For more information we refer the reader to [LV83] or [Vog83]. Our terminology chiefly follows the latter reference.

Let $\mathcal{D}$ denote the set of pairs $(\mathcal{O}, \gamma)$, where $\mathcal{O} \in K \backslash X$ and $\gamma$ is a $K$-equivariant local system on $\mathcal{O}$. The choice of $\gamma$ is equivalent to the choice of a character of the component group of the stabiliser $K_{x}$ of a point $x \in \mathcal{O}$. In particular, $\gamma$ is unique if $K_{x}$ is connected. Since $\mathcal{O}$ is determined by $\gamma$, we may abuse notation and write $\gamma$ for $(\mathcal{O}, \gamma)$. With each pair $\gamma, \delta \in \mathcal{D}$, we associate polynomials $R_{\gamma, \delta}, P_{\gamma, \delta} \in \mathbb{Z}[q]$. The $R$ polynomials can be computed using a recursive procedure which we refrain from stating in full generality here; see [Vog83, Lemma 6.8] for details. A special case sufficient for our purposes is formulated in Proposition 5.2 below.

Let $\mathcal{M}$ denote the free $\mathbb{Z}\left[q, q^{-1}\right]$ module with basis $\mathcal{D}$. For fixed $\delta \in \mathcal{D}$, we have in $\mathcal{M}$ the identity

$$
q^{-l(\delta)} \sum_{\gamma \leq \delta} P_{\gamma, \delta}(q) \gamma=\sum_{\beta \leq \gamma \leq \delta}(-1)^{l(\beta)-l(\gamma)} q^{-l(\gamma)} P_{\gamma, \delta}\left(q^{-1}\right) R_{\beta, \gamma}(q) \beta
$$

which subject to the restrictions $P_{\gamma, \gamma}=1$ and $\operatorname{deg}\left(P_{\gamma, \delta}\right) \leq(l(\delta)-l(\gamma)-1) / 2$ uniquely determines the Kazhdan-Lusztig-Vogan $(K L V)$ polynomials $P_{\gamma, \delta}$ [Vog83, Corollary 6.12] (i) Here, $l(\cdot)$ indicates the dimension of the corresponding orbit, and the order on $\mathcal{D}$ is the Bruhat $\mathcal{G}$-order [Vog83, Definition 5.8].

KLV polynomials serve as measures of the singularities of symmetric orbit closures; $c f$. [Vog83, Theorem 1.12]. In particular, their coefficients are nonnegative. Another consequence is the following:

Proposition 2.1 Let $\leq$ denote the order relation in $V$, i.e. containment among orbit closures. Given orbits $\mathcal{P}, \mathcal{O} \in K \backslash X$ with $\mathcal{P} \leq \mathcal{O}$, let $\delta=\left(\mathcal{O}, \mathbb{C}_{\mathcal{O}}\right)$, where $\mathbb{C}_{\mathcal{O}}$ is the trivial local system. Then, $\overline{\mathcal{O}}$ is rationally smooth at some (equivalently, every) point in $\mathcal{P}$ if and only if

$$
P_{\gamma, \delta}= \begin{cases}1 & \text { if } L=\mathbb{C}_{\mathcal{Q}} \\ 0 & \text { if } L \neq \mathbb{C}_{\mathcal{Q}}\end{cases}
$$

for all $\gamma=(\mathcal{Q}, L) \in \mathcal{D}$ with $\mathcal{P} \leq \mathcal{Q} \leq \mathcal{O}$.

The gadgets just described are fundamental ingredients in the representation theory of $G_{\mathbb{R}}$. More precisely, the KLV polynomials govern the transition between important families of $\left(\mathfrak{g}, K_{\mathbb{R}}\right)$-modules. See [LV83, Vog83] for more details.

\footnotetext{
(i) Note that there is a typo which has an impact on the cited result. We are grateful to D. A. Vogan for pointing out that the displayed formula in the statement of [Vog83 Lemma 6.8] should read
}

$$
D(\delta)=u^{-l(\delta)} \sum_{\gamma}(-1)^{l(\gamma)-l(\delta)} R_{\gamma, \delta}(u) \gamma .
$$




\section{Restricting the involution}

Consider the set $\mathcal{V}=\left\{g \in G \mid \theta\left(g^{-1}\right) g \in N\right\}$. The set of orbits $K \backslash \mathcal{V} / T$ parametrises $K \backslash X$. In this way, the map $\mathcal{V} \rightarrow W$ given by $g \mapsto \theta\left(g^{-1}\right) g T$ induces the map $\varphi: V \rightarrow W$ which was mentioned in the introduction. Observe that the image of $\varphi$ is contained in $\mathcal{I}(\theta)$.

Throughout this paper we shall only allow certain choices of $\theta$. More precisely, we from now on assume that $\theta$ obeys the following condition:

Hypothesis 3.1 The fixed point subgroup $K$ is connected. Moreover, $\varphi: V \rightarrow W$ satisfies $\varphi\left(v_{0}\right) \in \iota(\theta)$, where $v_{0} \in V$ is the maximum element, i.e. the dense orbit.

Remark 3.2 If $G$ is semisimple and simply connected, then $K$ is necessarily connected. This result is due to Steinberg [Ste68, Theorem 8.1]. In some sense, the general situation can be reduced to the study of semisimple simply connected $G$; see [RS90].

Several consequences are collected in the next proposition. For the proof, see [Hul09]. Let $\Phi$ denote the root system of $G, T$ and write $R \subset W$ for the corresponding set of reflections.

Proposition 3.3 Hypothesis 3.1 implies the following:

(i) The map $\varphi$ yields a poset isomorphism $V \rightarrow \operatorname{Br}(\iota(\theta))$.

(ii) There is a unique $K$-equivariant local system, namely $\mathbb{C}_{\mathcal{O}}$, on each orbit $\mathcal{O} \in K \backslash X$. In particular, the sets $\mathcal{D}, K \backslash X$ and $\iota(\theta)$ may be identified, and the Bruhat $\mathcal{G}$-order on $\mathcal{D}$ coincides with $V$ and $\operatorname{Br}(\iota(\theta))$.

(iii) Let $\alpha \in \Phi$ and denote by $G_{\alpha} \subseteq G$ the corresponding rank one semisimple group. Then, we are in one of the following two situations:

(a) The root $\alpha$ is compact imaginary. That is, $G_{\alpha} \subseteq K$.

(b) The root $\alpha$ is complex (meaning $\theta(\alpha) \neq \alpha$ ) and $\theta(\alpha)+\alpha \notin \Phi$.

(iv) If $r \in R$, then $\theta(r) r=r \theta(r)$.

(v) The poset $\operatorname{Br}(\iota(\theta))$ is graded with rank function $\rho$ being half the ordinary Coxeter length. Moreover, $\rho(w)=l\left(\mathcal{O}_{w}\right)-l\left(\mathcal{O}_{\text {id }}\right)$.

The following example allows us to consider many of our results as generalisations of statements about Schubert varieties.

Example 3.4 If $G^{\prime}$ is a connected reductive complex linear algebraic group and $G=G^{\prime} \times G^{\prime}$, the involution $\theta$ which interchanges the two factors makes $K$ the diagonal subgroup. In this case, $\iota(\theta)=\mathcal{I}(\theta)$, so Hypothesis 3.1 is satisfied. The poset $\operatorname{Br}(\iota(\theta))$ coincides with $\operatorname{Br}\left(W^{\prime}\right)$, where $W^{\prime}$ is the Weyl group of $G^{\prime}$. There is a one-to-one correspondence between $K$-orbits in $X$ and Schubert cells in the Bruhat decomposition of the flag variety of $G^{\prime}$ which preserves a lot of structure including the property of having rationally smooth closure at a given orbit. 
In addition to the setting in Example 3.4 there are a few more cases that satisfy Hypothesis 3.1 . They are denoted $A I I, D I I$ and $E I V$ in the classification of symmetric spaces $G_{\mathbb{R}} / K_{\mathbb{R}}$ given e.g. in Helgason [Hel78, (ii) The corresponding Weyl groups are $A_{2 n+1}, D_{n}$ and $E_{6}$, respectively, with $\theta$ in each case restricting to the Weyl group as the unique nontrivial Dynkin diagram involution. Types $D$ and $E$ could in principle be handled separately. In the former case, $\iota(\theta)$ has a very simple structure ( $c f$. [Hul08, proof of Theorem 5.2]), whereas the latter admits a brute force computation. Thus, the main substance lies in the $A_{2 n+1}$ case where $\operatorname{Br}(\iota(\theta))$ is an incarnation of the containments among closures of $\operatorname{Sp}_{2 n}(\mathbb{C})$ orbits in the flag variety $\mathrm{SL}_{2 n}(\mathbb{C}) / B$; see $[\mathrm{RS} 90$, Example 10.4] for a discussion of this case. Nevertheless, we have opted to keep our arguments type independent regarding all assertions that are valid in the full generality of Hypothesis 3.1. There are two reasons. First, the natural habitat for Theorems 5.7 and 5.8 is the general setting; no simplicity would be gained by formulating the arguments in type $A$ specific terminology. Second, we hope that the less specialised viewpoint shall prove suitable as point of departure for generalisations beyond Hypothesis 3.1 .

\section{4 "Bruhat graphs"}

Let $*$ denote the $\theta$-twisted right conjugation action of $W$ on itself, i.e. $u * w=\theta\left(w^{-1}\right) u w$ for $u, w \in W$. Then $\iota(\theta)$ is the orbit of the identity element id $\in W$.

Recall that $I_{w}=\{u \in \iota(\theta) \mid u \leq w\}$.

Definition 4.1 Given $w \in \iota(\theta)$, let $\mathrm{BG}(w)$ be the graph with vertex set $I_{w}$ and an edge $\{u, v\}$ whenever $u=v * t \neq v$ for some reflection $t \in R$.

Notice that $\operatorname{BG}(u)$ is an induced subgraph of $\mathrm{BG}(w)$ if $u \leq w$. See Figure 1 for an illustration.

We shall refer to graphs of the form BG $(w)$ as Bruhat graphs, because in the setting of Example 3.4 . they coincide with (undirected versions of) the ordinary Bruhat graphs in $W^{\prime}$ introduced by Dyer [Dye91].

Next, we list some useful properties of Bruhat graphs. The proofs rely on combinatorial considerations and results from [Dye91]; see [Hul09] for details.

Lemma 4.2 Let $w \in \iota(\theta)$ and $u, v \in I_{w}, u \neq v$. Write $u=\theta\left(x^{-1}\right) x$ for $x \in W$. The following are equivalent:

(i) $\{u, v\}$ is an edge in $\mathrm{BG}(w)$.

(ii) There are exactly two distinct reflections $t \in R$ such that $u * t=v$.

(iii) There are exactly two distinct reflections $t \in R$ such that $\theta\left(x^{-1}\right) \theta(t) t x=v$. If $t$ is one of these reflections, then $\theta(t)$ is the other.

Lemma 4.3 If $\{u, v\}$ is an edge in $\mathrm{BG}(w)$, then either $u<v$ or $v<u$. Furthermore, $v$ has exactly $\rho(v)$ neighbours $u$ such that $u<v$.

Combining the first part of Brion's [Bri99, Theorem 2.5] with part(iii) of Proposition 3.3 shows that the rank of a vertex $v=\theta\left(x^{-1}\right) x$ in $\operatorname{BG}(w)$ is at most half the number of complex reflections (i.e. reflections that correspond to complex roots) $t \in R$ such that $\theta\left(x^{-1}\right) \theta(t) t x \leq w$. By Lemma 4.2, this is precisely the degree of $v$ in $\operatorname{BG}(w)$. We thus have the following fact:

(ii) The "usual" construction of $D I I$ would yield $G=\mathrm{SO}_{2 n}(\mathbb{C}), K=\mathrm{S}\left(\mathrm{O}_{2 n-1}(\mathbb{C}) \times \mathrm{O}_{1}(\mathbb{C})\right) \cong \mathrm{O}_{2 n-1}(\mathbb{C})$ so that $K$ is disconnected. However, passing to the fundamental cover, we have $G=\operatorname{Spin}_{2 n}(\mathbb{C}), K=\operatorname{Spin}_{2 n-1}(\mathbb{C})$ in agreement with Hypothesis 3.1 


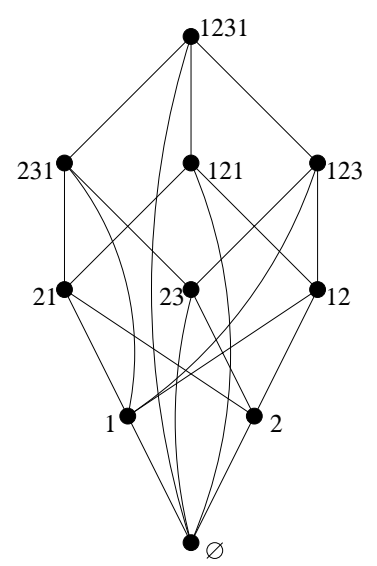

Fig. 1: A picture of the Bruhat graph BG( $w)$ where $w=s_{5} s_{3} s_{4} s_{5} s_{1} s_{2} s_{3} s_{1} \in \iota(\theta) \subset A_{5}$. Here, $s_{i}$ denotes the simple reflection $(i, i+1)$ in the usual manifestation of $A_{5}$ as the symmetric group $S_{6}$. The involution $\theta$ sends $s_{6-i}$ to $s_{i}$. A vertex $u \in I_{w}$ is labelled by the indices of a sequence of simple reflections whose product $x$ satisfies $u=\theta\left(x^{-1}\right) x$. The straight edges indicate the covering relation of $\operatorname{Br}(\iota(\theta))$.

Theorem 4.4 For $w \in \iota(\theta)$, the degree of each vertex in $\mathrm{BG}(w)$ is at least $\rho(w)$.

Remark 4.5 In the setting of Example 3.4 Theorem 4.4 specialises to "Deodhar's inequality" in $W^{\prime}$; see [BL00, §6] and the references cited there.

\section{A criterion for rational smoothness}

In general, the recursion for the $R$-polynomials mentioned in Section 2 is technically rather involved. Since we are assuming Hypothesis 3.1 however, the situation is simpler. Proposition 3.3 allows us to identify the indexing set $\mathcal{D}$ with $\iota(\theta)$. Rather than working with the actual $R$-polynomials as defined in [Vog83], we shall find it more convenient to use the following simple variation:

Definition 5.1 For $u, v \in \iota(\theta)$, let $Q_{u, v}(q)=(-q)^{\rho(v)-\rho(u)} R_{u, v}\left(q^{-1}\right)$.

With some labour, a combinatorially explicit recursion for the $Q_{u, v}$ can be extracted from the identity

$$
\sum_{u \in \iota(\theta)}(-1)^{\rho(u)} R_{u, w}(q) u=-\sum_{u \in \iota(\theta)}(-1)^{\rho(u)} R_{u, w * s}(q)\left(T_{s}+1-q\right) u ;
$$

see [Vog83, proof of Lemma 6.8]. The key is that the definition of the maps $T_{s}$ (see [Vog83, Definition 6.4]) simplifies a fair amount under Hypothesis 3.1 We refer the reader to [Hul09] for the details.

With $D_{\mathrm{R}}(v)$ denoting the descent set of $v \in \iota(\theta)$, i.e. the set of simple reflections $s$ such that $v s<v$, or equivalently $v * s<v$, the recursion takes the following form:

Proposition 5.2 For $s \in D_{\mathrm{R}}(v)$, we have

$$
Q_{u, v}(q)= \begin{cases}Q_{u * s, v * s}(q) & \text { if } u * s<u \\ q Q_{u * s, v * s}(q)+(q-1) Q_{u, v * s}(q) & \text { if } u * s>u \\ q Q_{u, v * s}(q) & \text { if } u * s=u\end{cases}
$$


Together with the "initial values" $Q_{u, u}(q)=1$ and $Q_{u, v}(q)=0$ if $u \not \leq v$, we may calculate any $Q_{u, v}$ using Proposition 5.2

In the setting of Example 3.4 , both the $R_{u, v}(q)$ and the $Q_{u, v}(q)$ coincide with the classical KazhdanLusztig $R$-polynomials introduced in [KL79]. The three lemmata coming up next hint that the $Q_{u, v}(q)$ may provide the more useful generalisation.

Lemma 5.3 For $u, v \in \iota(\theta)$, we have

$$
Q_{u, v}^{\prime}(1)= \begin{cases}1 & \text { if } u<v \text { and }\{u, v\} \text { is an edge in } \mathrm{BG}(v), \\ 0 & \text { otherwise. }\end{cases}
$$

Proof: Suppose $s \in D_{\mathrm{R}}(v)$. Differentiating the equation in Proposition 5.2 with respect to $q$, and using that $Q_{u, v}(1)=R_{u, v}(1)=\delta_{u, v}$ (Kronecker's delta), it follows that

$$
Q_{u, v}^{\prime}(1)=Q_{u * s, v * s}^{\prime}(1)+\delta_{u, v * s} .
$$

It is clear that $\{u * s, v * s\}$ is an edge in $\mathrm{BG}(v)$ if and only if the same is true about $\{u, v\}$. Employing induction on $\rho(v)$, it thus suffices to show that $u * s<v * s$ if $v * s \neq u<v$ and $\{u, v\}$ is an edge. Lemma 4.3 shows that $u * s$ and $v * s$ are comparable in this situation. The assertion $u * s>v * s$ would contradict the Lifting Property [Hul08, Lemma 2.7], and we are done.

Lemma 5.4 Denote by $\mu$ the Möbius function of $\operatorname{Br}(\iota(\theta))$. Then, $\mu(u, v)=Q_{u, v}(0)$ for all $u, v \in \iota(\theta)$.

Proof: Let us induct on $\rho(v)$. The assertion holds for $\rho(v)=0$ because $Q_{\text {id,id }}(q)=R_{\text {id,id }}(q)=1$. We shall demonstrate that $\mu(u, v)$ satisfies the recursion for $Q_{u, v}(0)$ derived from Proposition 5.2

Borrowing terminology from [Hul08], call $[u, v]$ full if every twisted involution in the interval $[u, v]$ is in fact a twisted identity. Combining Philip Hall's theorem (see e.g. [Sta97, Proposition 3.8.5]) with the topological results in [Hul08, Theorem 4.12] shows that

$$
\mu(u, v)= \begin{cases}(-1)^{\rho(v)-\rho(u)} & \text { if }[u, v] \text { is full, } \\ 0 & \text { otherwise }\end{cases}
$$

Pick $s \in D_{\mathrm{R}}(v)$. In case $u * s=u,[u, v]$ is not full, and $\mu(u, v)=0$ as desired. If $u * s>u$, it follows from [Hul08, Lemma 4.10] that $[u, v * s]$ is full if and only if $[u, v]$ is full. Thus, $\mu(u, v)=-\mu(u, v * s)$, and we are done. Finally, suppose $u * s<u$. If $[u * s, v * s]$ is full then $[u, v]$ is also full, again by [Hul08, Lemma 4.10]. On the other hand, [Hul08, Theorem 4.9] implies that $\mu(u * s, v)=-\mu(u, v)$, so if $[u * s, v * s]$ (and therefore $[u * s, v]$ ) is not full, then $[u, v]$ cannot be full either. Completing the proof, we conclude $\mu(u, v)=\mu(u * s, v * s)$.

Lemma 5.5 For all $v \in \iota(\theta)$,

$$
\sum_{u \leq v} Q_{u, v}(q)=q^{\rho(v)}
$$


Proof: We prove the lemma using induction on $\rho(v)$. Given $s \in D_{\mathrm{R}}(v)$, partition $I_{v}$ into three sets:

$$
A=\{u \leq v \mid u * s<u\}, B=\{u \leq v \mid u * s>u\}, C=\{u \leq v \mid u * s=u\} .
$$

By the Lifting Property [Hul08, Lemma 2.7], the map $u \mapsto u * s$ is a bijection between $A$ and $B$. The recursion in Proposition 5.2 therefore yields

$$
\begin{aligned}
\sum_{u \leq v} Q_{u, v}(q) & =\sum_{\substack{u \in A \\
u \leq v * s}} q Q_{u, v * s}(q)+\sum_{\substack{u \in B \\
u \leq v * s}}(1+q-1) Q_{u, v * s}(q)+\sum_{\substack{u \in C \\
u \leq v * s}} q Q_{u, v * s}(q) \\
& =q \sum_{u \leq v * s} Q_{u, v * s}(q)
\end{aligned}
$$

proving the claim.

Lemma 5.6 We have $P_{u, v}(0)=1$ whenever $u \leq v$ in $\iota(\theta)$.

Proof: The assertion is clear if $u=v$, and we employ induction on $\rho(v)-\rho(u)$.

Vogan's [Vog83, Corollary 6.12] translates to

$$
q^{\rho(v)-\rho(u)} P_{u, v}\left(q^{-1}\right)=\sum_{u \leq w \leq v} Q_{u, w}(q) P_{w, v}(q) .
$$

The left hand side is a polynomial with zero constant term. Hence, Lemma 5.4 implies

$$
P_{u, v}(0)=-\sum_{u<w \leq v} \mu(u, w)=\mu(u, u)=1 .
$$

We are finally in position to prove the main results. Since all necessary technical prerequisites have been established, the corresponding arguments from [Car94] can now be transferred to our setting more or less verbatim.

Theorem 5.7 Suppose $u, v \in \iota(\theta), u \leq w$. The following conditions are equivalent:

(i) The degree of $v$ in $\mathrm{BG}(w)$ is $\rho(w)$ for all $u \leq v \leq w$.

(ii) The KLV polynomials satisfy $P_{v, w}(q)=1$ for all $u \leq v \leq w$. That is, the orbit closure $\overline{\mathcal{O}_{w}}$ is rationally smooth at $\mathcal{O}_{u}$.

Proof: Define

$$
f_{u, w}(q)=q^{\rho(w)-\rho(u)}\left(P_{u, w}\left(q^{-2}\right)-1\right) .
$$

The $P$-polynomials have nonnegative coefficients. By Lemma 5.6, $f_{u, w}(q)$ too is a polynomial with nonnegative coefficients. Since it has vanishing constant term, $f_{u, w}^{\prime}(1)=0$ if and only if $f_{u, w}(q)=0$ which, in turn, is equivalent to $P_{u, w}(q)=1$.

Now,

$$
f_{u, w}^{\prime}(1)=(\rho(w)-\rho(u))\left(P_{u, w}(1)-1\right)-2 P_{u, w}^{\prime}(1) .
$$


Since $Q_{u, w}(1)=\delta_{u, w}$, we have

$$
\begin{aligned}
-2 P_{u, w}^{\prime}(1) & =\left.\frac{d}{d q} P_{u, w}\left(q^{-2}\right)\right|_{q=1} \\
& =2(\rho(u)-\rho(w)) P_{u, w}(1)+2 \sum_{u \leq v \leq w} Q_{u, v}^{\prime}(1) P_{v, w}(1)+2 P_{u, w}^{\prime}(1) .
\end{aligned}
$$

Hence,

$$
f_{u, w}^{\prime}(1)=\rho(u)-\rho(w)+\sum_{u \leq v \leq w} Q_{u, v}^{\prime}(1) P_{v, w}(1) .
$$

To begin with, assume (ii) holds. Then,

$$
\rho(w)-\rho(v)=\sum_{v \leq v^{\prime} \leq w} Q_{v, v^{\prime}}^{\prime}(1)
$$

for all $u \leq v \leq w$. Condition (i) now follows from Lemma 5.3 together with Lemma 4.3 .

Finally, let us prove (i) $\Rightarrow$ (ii) by induction on $\rho(w)-\rho(u)$. Suppose $u<v \leq w$ in $\operatorname{Br}(\iota(\theta))$. By Lemma 5.3 and the induction assumption, $Q_{u, v}^{\prime}(1) P_{v, w}(1)$ is one if $\{u, v\}$ is an edge in BG(w), zero otherwise. Since $\operatorname{deg}(u)=\rho(w), u$ has exactly $\rho(w)-\rho(u)$ neighbours $v$ such that $u<v$. We conclude $f_{u, w}^{\prime}(1)=0$ as desired.

Theorem 5.8 For $w \in \iota(\theta)$, the following are equivalent:

(i) For all $i$, $[\mathrm{id}, w]=\operatorname{Br}\left(I_{w}\right)$ has equally many elements of rank $i$ as of rank $\rho(w)-i$.

(ii) The graph $\mathrm{BG}(w)$ is regular.

(iii) $P_{u, w}(q)=1$ for all $u \leq w$.

Proof: (i) $\Rightarrow$ (ii) Let $n(i)$ denote the number of elements of rank $i$ in $[e, w]$. Now, using Lemma 4.3 and Theorem 4.4 , we count the edges in $\mathrm{BG}(w)$ in two ways and obtain

$$
\sum_{i=0}^{\rho(w)} n(i) i \geq \sum_{i=0}^{\rho(w)} n(i)(\rho(w)-i)
$$

with equality if and only if $\mathrm{BG}(w)$ is $\rho(w)$-regular. However, if $n(i)=n(\rho(w)-i)$ for all $i$, then equality does hold.

(ii) $\Rightarrow$ (iii) This follows from Theorem 5.7

(iii) $\Rightarrow$ (i) We claim that

$$
F_{w}(q)=\sum_{u \leq w} P_{u, w}(q) q^{\rho(u)}
$$

is a symmetric polynomial, i.e. $F_{w}(q)=q^{\rho(w)} F_{w}\left(q^{-1}\right)$. If the $P$-polynomials all are 1 , this means

$$
\sum_{u \leq w} q^{\rho(u)}=\sum_{u \leq w} q^{\rho(w)-\rho(u)} .
$$


It therefore remains to verify the claim. Observe that

$q^{\rho(w)} F_{w}\left(q^{-1}\right)=\sum_{u \leq w} q^{\rho(w)-\rho(u)} P_{u, w}\left(q^{-1}\right)=\sum_{u \leq w} \sum_{u \leq v \leq w} Q_{u, v}(q) P_{v, w}(q)=\sum_{v \leq w} P_{v, w}(q) \sum_{u \leq v} Q_{u, v}(q)$.

The claim now follows from Lemma 5.5 .

To illustrate these results, consider Figure 1] The interval [id, $w$ ] has three elements of rank three but only two of rank $\rho(w)-3=1$. By Theorem $\overline{5.8}, \overline{\mathcal{O}_{w}}$ is rationally singular. A more careful inspection of the graph shows that $s_{5} s_{1}$ and $e$ both have degree five whereas all other vertices have degree $\rho(w)=4$. By Theorem 5.7, the rationally singular locus of $\overline{\mathcal{O}_{w}}$ therefore is $\mathcal{O}_{s_{5} s_{1}} \cup \mathcal{O}_{e}$. Also, observe that the degree never decreases as we move down in the graph. This phenomenon is explained in the next section.

\section{Sufficiency of the bottom vertex}

In this final section, the criterion given in Theorem 5.7 is significantly improved in the special case $G=$ $\mathrm{SL}_{2 n}(\mathbb{C}), K=\mathrm{Sp}_{2 n}(\mathbb{C})$. In that case, as we shall see, whether or not an orbit closure $\overline{\mathcal{O}_{w}}$ is rationally smooth at $\mathcal{O}_{u}$ is determined by the degree of $u$ alone (Corollary 6.5 below). The corresponding statement for Schubert varieties is known to be true in type $A$ [De085] but false in general (see [BG03] for some elaboration on this). Necessarily, therefore, this section must be type specific since the results cannot possibly extend to the situation in Example 3.4 for arbitrary $G^{\prime}$.

We work in the set $F_{2 n}$ of fixed point free involutions on $\{1, \ldots, 2 n\}$. Let $\star$ denote the conjugation action from the right by the symmetric group $S_{2 n}$ on itself, i.e. $\sigma \star \pi=\pi^{-1} \sigma \pi$. Then, $F_{2 n}=w_{0} \star S_{2 n}$, where $w_{0}$ is the reverse permutation $i \mapsto 2 n+1-i$.

Let $\preceq$ denote the dual of the subposet of the Bruhat order on $S_{2 n}$ induced by $F_{2 n}$. The bottom element of this poset is $w_{0}$. Observe that if $u \neq u \star t$, then $u \star t \succ u$ iff $t$ is an inversion of $u$ (meaning $t=(a, b)$ with $a<b$ and $u(a)>u(b)$ ).

For $w \in F_{2 n}$, define the Bruhat graph $\mathrm{BG}(w)$ as the graph with vertex set $I_{w}=\left\{u \in F_{2 n} \mid u \preceq w\right\}$ and an edge $\{u, v\}$ whenever $u \neq v=u \star t$ for some transposition $t$. Thus, each edge has exactly two transpositions associated with it, and the graph is simple (no loops or multiple edges). If $w$ is understood from the context and $u \preceq w$, let $\mathcal{E}(u)$ denote the set of edges incident to $u$ in $\operatorname{BG}(w)$. Also, define $\operatorname{deg}(u)=|\mathcal{E}(u)|$.

Proposition 6.1 Suppose $W=A_{2 n-1} \cong S_{2 n}$ with $\theta: W \rightarrow W$ given by the unique nontrivial involution of the Dynkin diagram. Then, $x \mapsto w_{0} x$ defines a bijection $F_{2 n} \rightarrow \iota(\theta)$. Moreover, the bijection is an isomorphism of Bruhat graphs, i.e. $u \preceq w \Leftrightarrow w_{0} u \leq w_{0} w$ and $w_{0}(w \star t)=w_{0} w * t$.

Proof: This is immediate from the well known facts that $\theta(x)=w_{0} x w_{0}$ and that $x \mapsto w_{0} x$ is an antiautomorphism of $\operatorname{Br}(W)$.

Suppose $w \succeq u \neq w_{0}$ and let $r=(i, j), i<j$, be a transposition such that $u \star r \prec u$. Let $a=u(i)$ and $b=u(j)$. Thus, $a<b \neq i$.

For a transposition $t=(x, y)$, we use the notation $\operatorname{supp}(t)=\{x, y\}$.

Definition 6.2 Call a transposition $t$ compatible (with respect to $u$ and $r$ ) if either $\operatorname{supp}(t) \cap\{i, j\} \neq \emptyset$ or $\operatorname{supp}(t) \cap\{a, b, i, j\}=\emptyset$. 
Given an edge $e \in \mathcal{E}(u)$ there are precisely two transpositions $t$ and $t^{\prime} \neq t$ such that $e=\{u, u \star t\}=$ $\left\{u, u \star t^{\prime}\right\}$. At least one is compatible; let $t_{e}$ be such a one.

Definition 6.3 Given $e \in \mathcal{E}(u)$, define $\epsilon(e)=\left\{u \star r, u \star r \tau_{e}\right\}$, where

$$
\tau_{e}= \begin{cases}r t_{e} r & \text { if } u \star t_{e} r \preceq w, \\ t_{e} & \text { otherwise. }\end{cases}
$$

It is not a priori clear that $\epsilon(e)$ is independent of the choice of $t_{e}$, but this turns out to be the case. Here is the point of all this:

Theorem 6.4 Definition 6.3 defines an injective map $\epsilon: \mathcal{E}(u) \rightarrow \mathcal{E}(u \star r)$.

The proof of Theorem 6.4 hinges on combinatorial considerations revolving around the Standard Criterion characterising Bruhat order in symmetric groups; see e.g. [BBD05, Theorem 2.1.5]. The details can be found in [Hul09].

By Theorem 6.4 the degree can never decrease as we go down along edges in a Bruhat graph. In particular, if a vertex has the minimum possible degree, then so does every vertex above it:

Corollary 6.5 We have $\operatorname{deg}(v)=\operatorname{deg}(w)$ for all $u \preceq v \preceq w$ if and only if $\operatorname{deg}(u)=\operatorname{deg}(w)$.

Thus, to determine whether Condition (i) of Theorem 5.7 is satisfied, it suffices to check the degree of $u$.

Remark 6.6 The set $\mathcal{S}_{2 n}=\left\{w \in F_{2 n} \mid i \leq n \Rightarrow w(i) \geq n+1\right\}$ is in natural bijective correspondence with $S_{2 n}$ in a way which identifies $\operatorname{Br}\left(S_{2 n}\right)$ with $\preceq$. Restricted to $w \in \mathcal{S}_{2 n}$, Corollary 6.5 specialises to a result of Deodhar [De085] for type $A$ Schubert varieties. In that setting, our arguments are closely related to work of Billey and Warrington [BW03, §6]

Remark 6.7 Observe that for $G=\mathrm{SL}_{2 n}(\mathbb{C}), K=\mathrm{Sp}_{2 n}(\mathbb{C})$, Theorem 4.4 follows directly from Theorem 6.4. Thus, we have rederived Brion's [Bri99, Theorem 2.5] in this case.

\section{Acknowledgements}

The author is grateful to W. M. McGovern for many helpful discussions and to P. McNamara and E. Briand for translation assistance. Pardon my French.

\section{References}

[BL00] S. C. Billey and V. Lakshmibai, Singular loci of Schubert varieties, Progress in Mathematics 182 , Birkhäuser Boston, Inc., Boston, MA, 2000.

[BW03] S. C. Billey and G. S. Warrington, Maximal singular loci of Schubert varieties in $\mathrm{S} L(n) / B$, Trans. Amer. Math. Soc. 355 (2003), 3915-3945.

[BB05] A. Björner and F. Brenti, Combinatorics of Coxeter groups, Graduate Texts in Mathematics, Vol. 231, Springer-Verlag, New York, 2005. 
[BG03] B. D. Boe and W. Graham, A lookup conjecture for rational smoothness, Amer. J. Math. 125 (2003), 317-356.

[Bri99] M. Brion, Rational smoothness and fixed points of torus actions, Transform. Groups 4 (1999), $127-156$.

[Car94] J. B. Carrell, The Bruhat graph of a Coxeter group, a conjecture of Deodhar, and rational smoothness of Schubert varieties, Proc. Sympos. Pure Math. 56 (1994), 53-61.

[Deo85] V. V. Deodhar, Local Poincaré duality and nonsingularity of Schubert varieties, Comm. Algebra 13 (1985), 1379-1388.

[Dye91] M. Dyer, On the "Bruhat graph" of a Coxeter system, Comp. Math. 78 (1991), 185-191.

[Hel78] S. Helgason, Differential geometry, Lie groups, and symmetric spaces, Pure and Applied Mathematics 80, Academic Press, New York, 1978.

[Hul08] A. Hultman, Twisted identities in Coxeter groups, J. Algebraic Combin. 28 (2008), 313-332.

[Hul09] A. Hultman, Criteria for rational smoothness of some symmetric orbit closures, preprint 2009.

[KL79] D. Kazhdan and G. Lusztig, Representations of Coxeter groups and Hecke algebras, Invent. Math. 53 (1979), 165-184.

[LV83] G. Lusztig and D. A. Vogan, Jr., Singularities of closures of $K$-orbits on flag manifolds, Invent. Math. 71 (1983), 365-379.

[McG09] W. M. McGovern, Closures of $K$-orbits in the flag variety for $S U^{*}(2 n)$, preprint 2009.

[Ric82] R. W. Richardson, Orbits, invariants and representations associated to involutions of reductive groups, Invent. Math. 66 (1982), 287-312.

[RS90] R. W. Richardson and T. A. Springer, The Bruhat order on symmetric varieties, Geom. Dedicata 35 (1990), 389-436.

[RS94] R. W. Richardson and T. A. Springer, Complements to: The Bruhat order on symmetric varieties, Geom. Dedicata 49 (1994), 231-238.

[Spr85] T. A. Springer, Some results on algebraic groups with involutions, Advanced Studies in Pure Math. 6, 525-543, Kinokuniya/North-Holland, 1985.

[Spr94] T. A. Springer, A combinatorial result on $K$-orbits on a flag manifold, The Sophus Lie Memorial Conference (Oslo, 1992), Scand. Univ. Press, Oslo, 1994, 363-370.

[Sta97] R. P. Stanley, Enumerative combinatorics, vol. 1, Cambridge Univ. Press, 1997.

[Ste68] R. Steinberg, Endomorphisms of linear algebraic groups, Mem. Amer. Math. Soc. 80 (1968), 1108.

[Vog83] D. A. Vogan, Jr., Irreducible characters of semisimple Lie groups III. Proof of Kazhdan-Lusztig conjecture in the integral case, Invent. Math. 71 (1983), 381-417. 